\title{
DESCRIPCION DEL MACHO Y DE LA HEMBRA DE LUTZOMYIA LARENSIS N. SP. (DIPTERA: PSYCHODIDAE, PHLEBOTOMINAE) DEL ESTADO LARA, VENEZUELA
}

\author{
CONRADO ARREDONDO C.
}

Sección de Parasitología Médica de la Escuela de Medicina de la UCLA, Barquisimeto, Estado Lara, Venezuela

Description of the male and female of Lutzomyia larensis $\mathbf{n}$. sp. (Diptera: Psychodidae, Phlebotominae) from State of Lara, Venezuela $-A$ description is given of both the male and female of $a$ new species of phlebotomine sandfly caught in tree trunks in Yacambu National Park, State of Lara, Venezuela. The new species belongs to the subgenus Helcocyrtomyia, Barretto 1962 or group vexator, series peruensis of Theodor (1965) and of Young and Fairchild (1974). The male is characterized by the presence of 5 spines on the dististyle and a basal patch of long bristles on the basistyle, implanted on queratinized, strong tubercules. Female presents cibarial armature with 4 large teeth, and a segmented spermatheca similar to another species of the series peruensis.

Key words: taxonomy of sand flies - Lutzomyia larensis $\mathbf{n} . \mathbf{s p .}$

En capturas hechas por Rafael Urdaneta entre mayo y septiembre de 1985 en el Parque Nacional de Yacambú, Municipio Andrés Eloy Blanco y en Cubiro, Municipio Diego de Lozada del Estado Lara, fueron colectados entre especies conocidas, cinco machos y dos hembras de una especie, que se ha considerado como nueva y la cual hemos designado con el nombre de Lutzomyia larensis n.sp. como un homenaje a nuestro Estado Lara, región de Venezuela con una geografia arisca pero dotada de una fauna entomológica rica y excepcional.

\section{Lutzomyia larensis n.sp.}

Descripción del macho - Flebotomino grande midiendo 4.6 milímetros.

Cabeza midiendo 558 micra de largo, incluyendo el clipeo, por 472 de ancho. Clipeo midiendo 187 micra. Relación cabeza: clipeo 3,0: 1. Diámetro de los ojos 270 micra. Labroepifaringe con 450 micra a partir del borde an. terior del clipeo. Antenas con toro globoso, midiendo 86 micra de diámetro, presentando los demás segmentos las siguientes dimensiones en micra:

$$
\begin{array}{rrc}
\text { III }-690 & \text { VIII }-217 & \text { XIII }-142 \\
\text { IV }-285 & \text { IX }-195 & \text { XIV }-93 \\
\text { V }-262 & \text { X }-187 & \text { XV }-86 \\
\text { VI }-255 & \text { XI }-168 & \text { XVI }-82 \\
\text { VII }-236 & \text { XII }-153 &
\end{array}
$$

Relación A III/LE igual a 1,53:1. Ascoides llegando a la parte media del segmento. Palpos

Recebido el 25 de Noviembre de 1986. Aceptado el 22 de Deciembre de 1986. midiendo 1.124 micra siendo las medidas de los artículos las siguientes en micra: $19-71 ; 2{ }^{\circ}-$ $206,39-255 ; 49-131$ y $50-461$. La formula palpal es 1.4.2.3.5, siendo el 59 artículo mas largo que la suma del $30^{\circ}$ y el 40 .

Tórax midiendo 855 micra, desde el borde anterior del mesonoto al borde posterior del escutelo. El mesonoto y escutelo de color gris oscuro, post-escutelo y pleuras gris claro. Alas largas y anchas midiendo 3.510 micra de largo por 1.095 de ancho siendo la relación largo/ancho igual a 3,2:1. Las distancias alares principales son en micra: alfa - 1.200 ; beta - 345; gama 900 ; delta -570 . La relación alfa/beta es 3,47 : 1 , siendo beta menos que gama y delta. Patas, tienen en micra las siguientes dimensiones:

\begin{tabular}{lrrrr}
\hline & & Anterior & Média & Posterior \\
\hline Femur - & 1350 & 1215 & 1395 \\
Tibia - & 2250 & 2504 & 2895 \\
$\mathrm{~T}_{1}-$ & 1680 & 1725 & 1905 \\
$\mathrm{~T}_{2}-$ & 525 & 570 & 600 \\
$\mathrm{~T}_{3}-$ & 375 & 380 & 420 \\
$\mathrm{~T}_{4}-$ & 270 & 300 & 315 \\
$\mathrm{~T}_{5}-$ & 150 & 165 & 180 \\
\hline
\end{tabular}

Abdomen midiendo 3331 micra incluyendo el basistilo. Genitalia: basistilo mide 461 micra de largo por 120 micra de anchura máxima, presentando en la región basal un mechón de 20 a 24 cerdas ligeramente onduladas, que se implantan en tubérculos unidos, fuertes y queratinizados. Dististilo con 288 micra de largo por 41 micra de anchura máxima, presentando 5 espinas, con dos apicales fuertes implantadas al mis- 
mo nivel, dos ubicadas un poco mas arriba de la mitad del dististilo, que se insertan a un mismo nivel y una intermedia que se encuentra mas cercana a las espinas apicales. Parameros, midiendo 262 micra de largo por 86 micra de ancho en la base que es de forma rectangular, lue. go disminuye de diámetro en la región media, que mide 51 micra de anchura, presentando la mitad distal adelgazada, midiendo 22 micra de anchura en el ápice. La mitad distal del paramero presenta en la parte superior cerdas alargadas. Lóbulos laterales ligeramente engrossados midiendo 375 micra de largo por 37 de ancho. $L a$ melas submedianas normales. Bomba eyaculadora con 139 micra de largo. Ductos eyaculadores delgados de paredes lisas, midiendo 473 micra, siendo 3,4 veces mas largos que la bomba. Edeago, cónico, delgado midiendo 93 micra de largo por 22 de ancho en la base.

Descripción de la hembra ... Es grande, midiendo 4,4 milímetros. Cabeza, midiendo 600 micra de largo incluyendo el clipeo, por $510 \mathrm{de}$ ancho. Clipeo con 243 micra. Relación cabeza/ clipeo 2,46:1. Diámetro de los ojos 292 micra. Labro-epifaringe con 538 micra, a partir del borde anterior del clipeo. Antenas con toro globoso con 93 micra de diámetro, los demas seg. mentos presentando las siguientes dimensiones en micra:

$$
\begin{array}{rrr}
\text { III }-660 & \text { VII }-255 & \text { XI }-191 \\
\text { IV }-285 & \text { VIII }-232 & \text { XII }-183 \\
\text { V }-270 & \text { IX }-221 & \text { XIII }-172 \\
\text { VI }-255 & \text { X }-206 & \text { XIV }-134 \\
& & \text { XV }-101 \\
& & \text { XVI }-82
\end{array}
$$

Relación AIII/LE igual a 1,22:1. Ascoides simples cortos. Palpos midiendo 1309 micra, siendo las dimensiones en micra de los demas segmentos, las siguientes: $19-86 ; 20-285$; $30-300 ; 40-150$ y 50 - -487 . La formula palpal es 1 4.2.3.5 siendo el 5 artículo mayor que el 30. Cibario con 4 dientes horizontales equidistantes bien desarrollados con una hilera

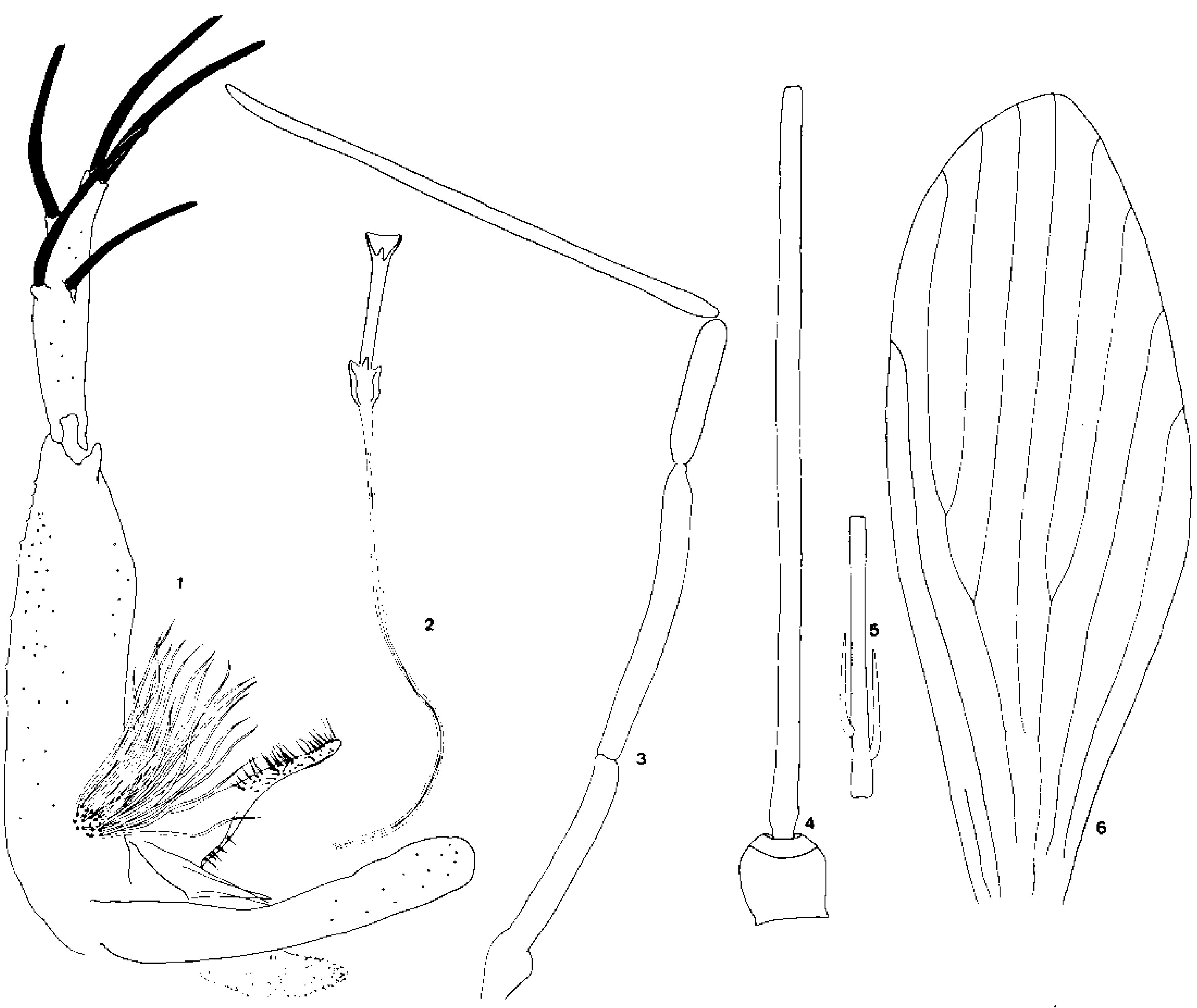

Lutzomyia larensis n.sp. - Holótipo macho - lamina número 954 - Fig. 1: genitália (x 400). Fig. 2: bomba e ductos eyaculadores (x 400). Fig. 3: palpo (x 400). Fig. 4: antena (toro e 30 segmento) (x 400). Fig. 5: antena (50 segmento) (x 400). Fig. 6: ala (x 100). 
irregular de dientes verticales. Area pigmentada poco visible, arco esclerotinizado incompleto.

Torax midiendo 960 micra desde el borde anterior del mesonoto al posterior del escutelo, mesonoto y escutelo gris oscuro, post-escutelo y pleuras gris claras. Alas largas y anchas, midiendo 3350 micra de lárgo por 1200 de ancho, siendo la relación largo/ancho igual a 2,79:1. Las distancias alares mas importantes son en micra: alfa -1.155 ; beta -405 ; gama $-975 \mathrm{y}$ delta -570 siendo la relación alfa/beta igual a 2,85:1. Las patas presentan las medidas siguientes en micra:

\begin{tabular}{|c|c|c|c|c|}
\hline & & Primer par & Segundo par & Tercer par \\
\hline Femur & - & 1410 & 1295 & 1470 \\
\hline Tibia & - & 2010 & 2310 & 2680 \\
\hline $\mathrm{T}_{1}$ & - & 1540 & 1590 & - \\
\hline $\mathrm{T}_{2}$ & - & 510 & 555 & - \\
\hline $\mathrm{T}_{3}$ & - & 375 & 388 & - \\
\hline$T_{4}$ & - & 300 & 300 & - \\
\hline $\mathrm{T}_{5}$ & - & 180 & 180 & - \\
\hline
\end{tabular}

Abdomen midiendo 2922 micra. Espermatecas segmentadas, alargadas, con 18 anillos, midiendo 60 micra de largo por 11 micra de ancho. Ductos individuales alargados y delgados midiendo 128 micra de longitud.

Localidad tipo - Parque Nacional de Yacambú, Município Andres Eloy Blanco, Estado Lara, Venezuela.

Tipos - Holotipo macho capturado en tronco de árbol a una altura de 1405 metros el Parque Nacional de Yacambú el 3-5 de 1985 por Rafael Urdaneta y depositado con el número 954 en la Sección de Parasitología Médica de la Escuela de Medicina de la Universidad Centro Occidental - "Lisandro Alvarado", Barquisimeto, Venezuela.

Alotipo - Capturado en tronco de árbol a una altura de 1405 metros en el Parque Nacional de Yacambú el 17-9 de 1985 por Rafael Urdaneta y depositado con el número 1.032 en la Sección de Parasitología Médica de la Escuela de Medicina de la Universidad Centro Occi.
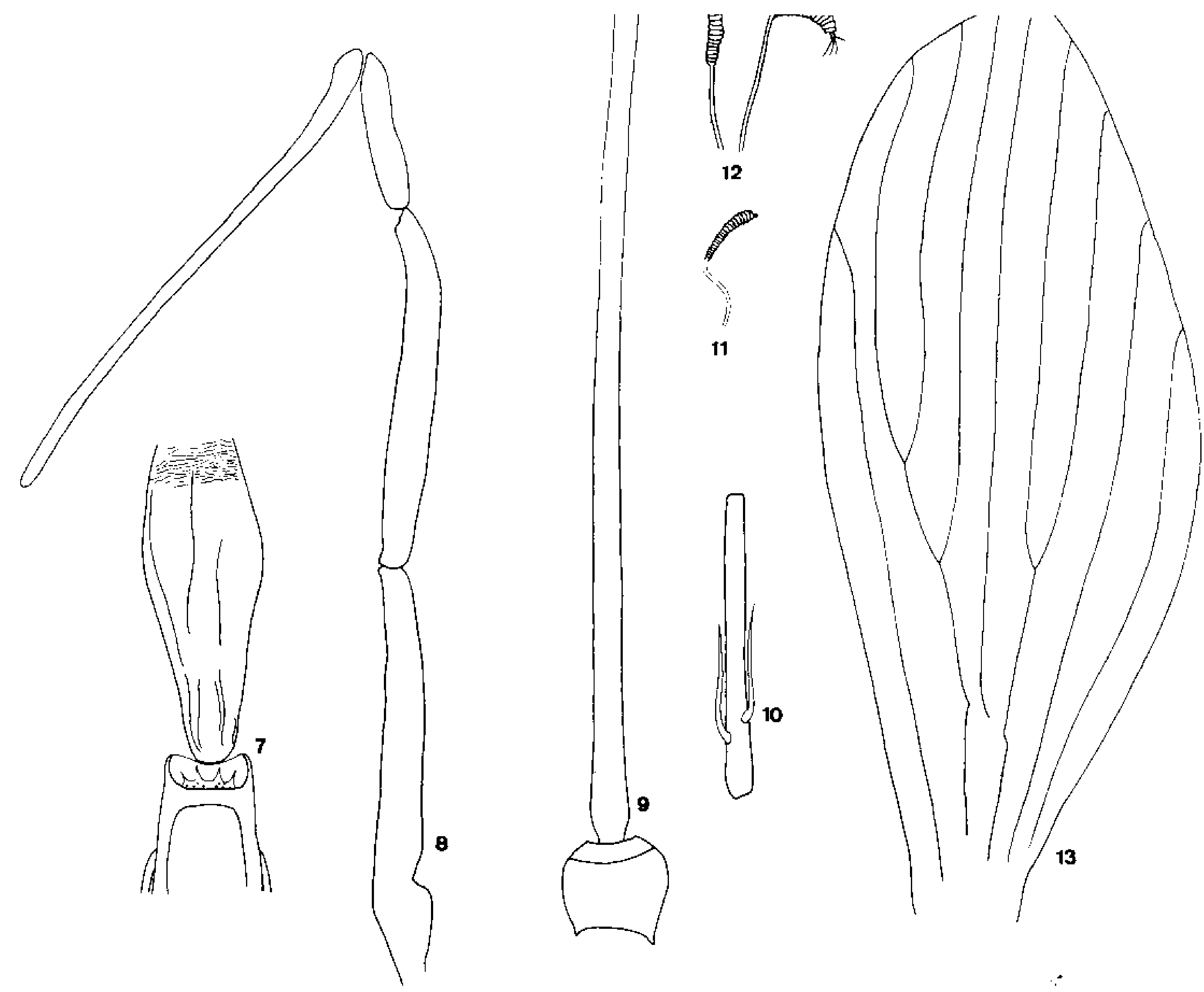

Lutzomyia larensis n.sp. - Alótipo hembra - lamina número 1032 - Fig. 7: cibário (x 400). Fig. 8: palpo (x 400). Fig. 9: antena (toro e 3\% segmento (x 400). Fig. 10: antena (50 segmento) (x 400). Fig. 11: espermateca (x 400). Fig. 12: espermatecas (x 600). Fig. 13: ala (x 100). 
dental "Lisandro Alvarado", Barquisimeto, Venezuela.

Paratipos - 15 y 19 , capturados por Rafael Urdaneta en tronco de árbol a 1350 metros de altura, en Cubiro, Municipio Diego de Lozada, Estado Lara, Venezuela el 10-7 de 1985 y depositado con los números 986 y 987 en la Sección de Parasitología Médica de la Escuela de Medicina. $30^{\circ}$ capturados en el Parque Nacional de Yacambú, Estado Lara a 1.400 metros de altura el 17 y $18-9$ de 1985 , dos depositados con los números 1.033 y 1.034 en la Sección de Parasitología Médica de la Escuela de Medicina de la Universidad Centro Occidental "Lisandro Alvarado", Barquisimeto.

Cometarios - Lutzomyia larensis $\mathrm{n}$. $\mathrm{sp}$. debe ser incluida en el sub-género Helcocyrtomyia Barretto 1962, grupo vexator serie peruensis de Theodor (1965) y de Young y Fairchild (1974).

El macho se identifica con facilidad por 1a forma del paramero, que presenta una región basal ancha de forma rectangular y por el mechón de cerdas del basistilo, formado por 20.24 cerdas, implantadas en tubérculos gruesos y queratinizados. Lutzomyia ceferinoi (Ortiz \& Alvarez, 1963a, b) es la especie que mas se le parece, pero posee el paramero cilindrico y un mechón de cerdas en el basistilo formado por 12 a 14 cerdas.

La hembra posee espermatecas de tamaño muy reducido y otras características próximas a las de otras especies del mismo grupo.

El macho y la hembra de $L$. larensis se correlacionan entre si por su similitud en tamaño, coloración $\mathrm{y}$ por haber sido colectadas en los mismos biotopos en diferentes ocasiones.

\section{RESUMEN}

Descripción del macho y de la hembra de Lutzomya larensis n. sp. (Diptera: Psychodidae, Phlebotominae) del Estado Lara, Venezuela - Es dada 1a descripción del macho y de la hembra de una nueva espécie de flebotomíneo recolectado en tronco de árboles en el Parque Nacional de Yacambú, Estado Lara, Venezuela.

La nueva espécie pertenece al subgénero Helcocyrtomyia, Barretto, 1962 o al grupo vexa. tor, série peruensis de Theodor (1965) y de Young \& Fairchild (1974). El macho se identifica por la preséncia de 5 espinos en el dististilo y por el mechón de cerdas largas en el basistilo, implantadas en tubérculos gruesos y queratini- zados. La hembra a la vez presenta armadura del cibário con 4 dientes largos y una espermateca segmentada semejante a las de otras espécies de la série peruensis.

\section{Palabras-claves: taxonomia de flebotominos - Lutzomyia larensis $\mathrm{n}$. $\mathrm{sp}$.}

\section{RESUMO}

Descrição do macho e da fêmea de Lutzomyia larensis n. sp. (Diptera: Psychodidae, Phlebotominae) do Estado Lara, Venezuela - $\hat{E}$ apresentada a descrição do macho e da fêmea de uma nova espécie de flebotomíneo cole tado em tronco de árvore no Parque Nacional de Yacambú, Estado Lara, Venezuela.

A nova espécie pertence ao subgênero $\mathrm{Hel}$. cocyrtomyia, Barretto, 1962, ou grupo vexator, série peruensis de Theodor (1965) e de Young \& Fairchild (1974). O macho da nova espécie apresenta cinco espinhos no dististilo e no basistilo um grupo de cerdas longas, implantadas em tubérculos fortes e queratinizados e a fêmea mostra quatro dentes horizontais no cibário e espermatecas segmentadas, semelhantes ao de outras espécies da série peruensis.

Palavras-chave: taxonomia de

flebotomíneos - Lutzomyia larensis n. sp.

\section{AGRADECIMIENTOS}

Muchas gracias ao Dr. Edelberto Santos Dias, del Centro de Pesquisas René Rachou, FIOCRUZ, por los magnificos dibujos.

\section{REFERENCIAS}

BARRETTO, M.P., 1962. Novos subgêneros de Lutzom via França, 1924 (Diptera, Psychodidae, subfam ília Phlebotominae) Rev. Inst. Med. trop. Säo Paulo, $4: 91 \cdot 100$.

ORTIZ, I. \& ALVAREZ, A., 1963a. Sobre los Phlebotomus Americanos de cinco espinas con la descripción de una nueva especie (Phlebotomus ceferinoi sp. nov.) de Venezuela (Diptera: Psychodidae). Rev. Venez. San. y Assist. Soc., 28 (3) :311-314.

ORTIZ, I. \& ALVAREZ, A., 1963b. Sobre un nuevo Phlebotomus (P. ceferinoi nov. sp.) de Venezuela (Insecta: Diptera, Psychodidae). Sal. Pub., $5: 285$ 288.

THEODOR, O., 1965. On the classification of American Phlebotominae. J. Med. Entomol, 2 :171197.

YOUNG, D.G. \& FAIRCHILD, G.B., 1974 - Studies of Phlebotomine sand flies. Gainesville: Department of Entomology and Nematology, University of Florida -151 p. 